\title{
The magnitude and associated Factors of Postoperative Trichiasis among adults who underwent Trachomatous Trichiasis Surgery in Ambassel District, North-East Ethiopia
}

\author{
Abdu Tabor Yimam \\ Ambasel District Health Office \\ Gizachew Tadesse Wassie ( $\square$ leulgzat@gmail.com ) \\ Bahir Dar University https://orcid.org/0000-0001-6213-0191 \\ Getu Degu Alene \\ Bahir Dar University College of Medical and Health Sciences
}

Research article

Keywords: Trachomatous, Trichiasis, Recurrence, Magnitude, Ambassel, Ethiopia

Posted Date: September 18th, 2020

DOI: https://doi.org/10.21203/rs.3.rs-61501/v1

License: (c) (i) This work is licensed under a Creative Commons Attribution 4.0 International License. Read Full License 


\section{Abstract \\ Background}

In Trachoma endemic countries, many people who underwent Trichiasis surgery faced a recurrence of the disease. Postoperative Trichiasis is a significant problem for patients and health care providers because it puts the eye at renewed risk of sight loss. Despite the low utilization of Trachomatous Trichiasis (TT) surgery and high recurrence rate, evidences that elucidate why TT recurs after surgery are limited. This study was aimed to assess the magnitude and associated factors of postoperative Trichiasis among 18 years and above individuals who underwent Trachomatous Trichiasis surgery between 2006 and 2011 Ethiopian Fiscal year in Ambassel District, North-East Ethiopia, 2020.

\section{Methods}

The community-based cross-sectional study design was conducted from March 10 to March 23/2020 in selected kebeles of Ambassel District. The required sample size was calculated using EPI-INFO Version 7. A multi-stage sampling technique was used to employ a total of 506 individuals. Data were collected through the intervieweradministered structured pre-tested questionnaire and entered into EpiData version 3.1 and then exported to SPSS version 23.0. Bi-variable and multivariable logistic regression models were fitted to identify associated factors of Trachomatous Trichiasis recurrence.

\section{Results}

Four hundred ninety two individuals participated in this study with response rate of $97.2 \%$. In Ambassel district the prevalence of postoperative Trichiasis was $23.8 \%(95 \% \mathrm{Cl}=19.9-27.8)$. Among associated factors of postoperative Trichiasis age 50-59 AOR = 3.34 $(\mathrm{Cl}=1.38-8.1), 60-69 \mathrm{AOR}=3.24(\mathrm{Cl}=1.38-7.61), \geq 70$ years $\mathrm{AOR}=6.04(\mathrm{Cl}=2.23-$ 16.41), duration since surgery $A O R=1.7(C l=1.35-2.14)$, complication $A O R=2.98(C l=1.24-7.2)$, washing the face two times $A O R=0.25(\mathrm{Cl}=0.13-0.47)$, washing the face three and more times $A O R=0.1(\mathrm{Cl}=0.41-0.25)$, taking Azithromycin following surgery $A O R=0.19(\mathrm{Cl}=0.09-0.41)$, pre-operative epilation history $A O R=2.11,(\mathrm{Cl}=1.14$, 3.9)and having a knowledge about TrachomaTtrichiasis $A O R=0.21(\mathrm{Cl}=0.08-0.58)$ showed a statistical significant association.

\section{Conclusions}

The prevalence of postoperative Trichiasis in Ambassel District was higher than most Ethiopian studies. Age, frequency of face washing, medication following surgery, duration since the last surgery, knowledge about trachoma, pre-operative epilation history and complication after surgery were identified to be independent factors. To minimize postoperative Trichiasis stakeholders need to consider health education for patients, provision of Azithromycin after surgery and proper training for IECWs.

\section{Background}

Trachoma is one of the leading of causes Trachiasis caused by Chlamydia Trachomatous and transmitted through contact with the eye discharge from the infected person(1). 
Postoperative Trichiasis (POT) is defined as when Trichiasis (one or more lashes touching the globe) is found again in an operated eye of a patient after surgery(2).Recurrence of Trichiasis following intervention is mentioned as a major barrier for the better uptake of TT surgery $(3,4)$.

Based on the WHO grading system for trachoma, the disease is classified into five grades. These are Trachomatous Inflammation-Follicular (TF), the presence of five or more follicles, it mostly requires topical treatment; Trachomatous Inflammation-Intense ( $\mathrm{TI})$, pronounced inflammatory thinking of conjunctiva, which topical and systemic treatments are considered; Trachomatous Scarring (TS) - when scars are visible as in the tarsal conjunctiva and which may obscure Tarsal blood vessels; Trachomatous Trichiasis (TT) - when an individual is referred for eyelid surgery; and Corneal Opacity (CO) - a stage during which a person is irreversibly blind(5).

In Ethiopia, about 173,945 (60\% of the plan) persons operated and 39,339,311 (63\% of the plan) Doses of Azithromycin distributed during mass drug administration in 2017. In the Amhara region 91,977 persons under went the surgery, 13,651,377 doses of Azithromycin were distributed during mass drug administration; all (3459) villages with health education and 1,802,962 $\mathrm{HH}$ have latrine facilities in 2017 (6).

Globally, over 40 million people are estimated to suffer from active trachoma, and about 8.2 million have to Trichiasis; 2.2 million have a visual impairment and 1.2 million of these are irreversibly blinded from trachoma(7, 8). Together with Trichiasis, the economic loss due to loss of productivity from blindness and visual impairment is high (9).

Postoperative Trichiasis has a negative impact on the provision of good quality service and acceptability of Trichiasis surgery outcome; and this may affect the progression of eliminating trachoma. Individuals with Trichiasis are less likely to receive surgery when they see people who have not been treated better (10). And also, it is a significant problem because re-operation has a higher risk of surgical failure than a primary operation and the eye may face the risk of blindness. Studies suggest that Re-operation has up to 13-fold increased risk of developing postoperative Trichiasis (11).

Unfortunately, the rate of postoperative Trichiasis after TT surgery is unacceptably high in many countries. A survey that assessed POT in Africa found that the rate was ranged from $2.3 \%$ at 6 weeks to $65 \%$ at 7 years(12).

Despite the low utilization of TT surgery, there are limited researches that elucidate why TT recurs following surgery. The studies on postoperative Trichiasis undertaken in Ethiopia are not only few in number but also narrow and limited in towns.

This study was aimed at assessing the magnitude of postoperative Trichiasis and identifying associated factors of postoperative Trichiasis among 18 years and above individuals who underwent TT surgery between 2006 and 2011 EFY in the study area.

\section{Methods}

\section{Study area}

This study was carried out in Ambassel district, South Wollo Administrative Zone, Amhara Region North East Ethiopia. Ambassel is one of the 22 districts in the South Wollo administrative zone which is found in Amhara regional state. The district capital Wuchale is located $60 \mathrm{~km}$ far from Dessie town (the capital city of South Wollo Administrative zone), $460 \mathrm{Km}$ to the North-East direction from Addis Ababa (the capital city of Ethiopia). The district is established with one urban and 23 rural Kebeles. All kebeles are trachoma endemic areas. In 2019, the district population was estimated to be 143,519 of which 66,039 (46 \%) were females. The district has seven Health Centers, 23 rural and one 
urban health Posts. The district health office provided Trachomatous Trichiasis surgery for 2133 individuals between 2013 and 2019. An estimated 831 backlog TT cases are available in the district (34) (Figure 1).

\section{Study design and period}

This community-based cross-sectional study design was conducted among selected kebeles of Ambassel district from March 10 to March 23, 2020.

\section{The source population}

The source population included all 18years old and above individuals who underwent Trachomatous Trichiasis surgery from 20013 to 2019 and living in all kebeles of Ambassel district.

\section{Study population}

All 18 years and above individuals who underwent Trachomatous Trichiasis surgery from 2013 to 2019 EFY living in selected kebeles of Ambassel district.

\section{Inclusion and exclusion criteria}

\section{Inclusion criteria}

Any individual 18 years and above who had undergone Trachomatous Trichiasis surgery from 2013 to 2019 EFY and residing in the study area were included.

\section{Exclusion criteria}

Persons who were seriously ill and unable to respond to the question during data collecting time were excluded.

\section{Sample size determination}

Objective one: previously there was no recent prevalence study on Trachomatous Trichiasis recurrence. So considering $50 \%$ postoperative Trichiasis prevalence rate, $95 \%$ confidence level, and 5\% margin of error; the expected sample size was calculated using the STAT CALC program of EPI-INFO Version 7 computer software statistical package. The study subjects were selected through by passing more than one stage and the design effect was taken to be 1.5.

$Z=1.96$ (Standard normal deviate corresponding to $95 \%$ confidence interval)

$\mathrm{p}=0.50=$ (estimated Trachomatous Trichiasis recurrence)

$q=0.50$ (Complimentary probability $(1-p) ; 1-0.5)$

$E=0.05$ (Desired level of accuracy) 


$$
\begin{aligned}
& \text { Design Effect }=1.5 \\
& n=\frac{(z \alpha / 2)^{2 *} P(1-P)}{d^{2}} *(D E) \\
& n=\frac{1.96 * 1.96 * 0.50(1-0.50)}{0.05^{2}} *(\text { DesignEffect }) \\
& \mathrm{n}=3.8416 * 0.25 / 0.0025 *(1.5) \\
& \mathrm{n}=0.9604 / 0.0025 *(1.5) \\
& \mathbf{n}=\mathbf{5 7 6}
\end{aligned}
$$

Objective two: The sample size was calculated using EPI-INFO version 7 by different variables from different literature which have a significant association with the outcome variable considering the assumption of $95 \% \mathrm{Cl}, 1.1$ case to control ratio, $80 \%$ power, \% of outcome in unexposed group and \% of outcome in exposed group or odds ratio. Accordingly, the sample size estimated was 585.

Based on the above calculations, 585 was taken as the final sample size as it was slightly greater than 576 .

The sample size determined above was for a large target population exceeding 10, 000 and some correction was required. According to the data obtained from the district, the total individuals who underwent Trachomatous Trichiasis surgery from 2013 to 2019 in the district were 2133.

$$
\text { The corrected sample size was: } \quad \mathbf{N}=\frac{n}{1+\frac{n}{N}}=\frac{585}{1+\frac{585}{2133}}=460
$$

Finally, including 10\% non-response rate (46 individuals), the total sample size of the study was 506 individuals.

\section{Sampling Technique and procedures}

A multistage sampling technique was used. In the district, from 24 total kebeles one urban kebele and seven rural kebeles were selected by the lottery method. A sampling frame or lists of individuals who underwent TT surgery are available in the district office and at the kebele level. For each kebele, individuals with 18 years and above who underwent TT surgery were selected from their list using computer generated simple random sampling method. SPSS version 23 computer software program was used to select individuals to be included in the sample and the number of study participants for each Kebele was determined by proportional allocation based on the number of individuals who had TT surgery. Repeated visits were made in situations when individuals who were supposed to be respondents were not present during the initial attempt. The schematic presentation of sampling is shown in Figure 2.

\section{Data collection tools and procedures}

Interviewer administered structured questionnaire was used to collect information on socio-demographic and household characteristics, knowledge about trachoma, medical and surgical factors and environmental factors from selected individuals. The questionnaire also contains an observational checklist containing variables such as presence 
or absence of inverted one or more eyelash, presence of Trachomatous infection, surgeon's code who performed the procedure.

The data was collected by Integrated Eye Care Workers (IECWs) who had not performed surgery on any of the study communities and were blinded about which surgeon had conducted the surgery.

The data collectors examined the eyes and recorded the findings for the eye. Ophthalmic loupe with a magnification of x2.5 and torchlight was used for this assessment. Also, Checklist was used to collect information from TT surgery log book for variables like surgeon code, type of surgery, post-surgical treatment and eyelid operated. Participants who have any abnormalities diagnosed, the data collector referred the individual to the nearest health facility for eye care according to national guidelines and 159 individuals who had active trachoma on eye examination were provided Tetracycline eye ointment.

\section{Variables}

\section{Dependent variable}

Postoperative Trichiasis (present or absent)

\section{Independent variables}

Economic status: Wealth index

Socio-demographic: Sex, age, religion, family size, having < 10 years old children at home, place of residence, level of education, occupation

Personal factors: Knowledge about trachoma, Face washing practice

Environmental factors: Utilization of toilet, Water access

Medical and surgical factors: Surgeon variability, post-surgical treatment, lid surgery in the past, history of epilation, eye lid operated, post-operative infection of the eye, choice of surgical procedure

\section{Operational definitions}

Postoperative Trichiasis: A person presenting with one or more eyelashes touching the globe or history of epilation after surgery(54).

The respondent who had inflammation on the eye or more than five follicles on the tarsal conjunctiva during data collection time was taken as having Trachoma.

Epilation: When an individual removing in turned eyelashes mechanically from the eyes by local device like "worento".

Integrated Eye Care Workers (IECWs): Nurse or health officers who are trained for one month in primary eye care including TT surgery that makes operation from the study participants.

Clean face - An individual who did not have an eye discharge or nasal discharge on face at the time of survey.

Water access: - The person reported on how long it took for a return journey to collect water for domestic use from the main water source, including time spent in the queue. 
Improved Latrine: A toilet which was constructed by simply obtained local material but it has a fully covered floor, wall, and roof, the well is long to have stood, the opening has cover and hand washing facility.

Toilet utilization: - The utilization of latrine by family member that was confirmed by asking the study participants and visual inspection

Post-surgical treatment: Oral azithromycin or TTC eye ointment treatment given for patients who had got Trachomatous Trichiasis surgery

Knowledge about trachoma: Knowledge questions were calculated after some questions were presented. Correct answers were given score 1 and incorrect answers 0 . Then the sum was computed and those who scored $50 \%$ and above were labeled as "good knowledge" and those who scored below 50\% were labeled as "poor knowledge".

Wealth index: It was assessed by including domestic animals, agricultural land and commodity that family members used, finally calculated using SPSS. When the wealth index, was $<20 \%$ it was labeled as poorest, $20-40 \%$ poor, $41-60 \%$ middle income, $61-80 \%$ rich, and greater than $80 \%$ richest.

\section{Data quality assurance}

To maintain the quality of the data a pre-tested structured questionnaire was used to collect data. The pre-test was done in a nearby district rural community and an appropriate correction was taken to the questionnaire. Adequate training was provided for the data collectors and supervisor for two days on how to conduct the interview, how to approach the respondents and perform the clinical examination. On-site supervision and feedback was given to data collectors. Data entry was done by the principal investigator after checking for completeness and coding.

\section{Data processing and analysis}

The collected data were checked for completeness and entered into EpiData version 3.1 software programs and then exported to SPSS version 23 software programs for data cleaning and analysis. Bi-variable logistic analysis was performed to see an association of each independent variable with the Trachomatous Trichiasis recurrence. Explanatory variables with P-value $\leq 0.2$ were selected as candidates for the final model. Binary logistic regression model (multivariable analysis) was performed to assess the association between the independent variables and the outcome variable. The level of significance was set at $95 \% \mathrm{Cl}(p<0.05)$ for statistical significance in the model. To assess the model fitness for the application of multivariable logistic regressions, Hosmer-Lemeshow goodness-of-fit test statistic was employed.

\section{Results}

\section{Socio-demographic characteristics of study participants}

The response rate of the study was $97.2 \%$. Of the non-respondents, two of them were unable to respond due to illness, three were not present after three times visit, seven of them left to other districts and the remaining two died due to other causes. The age distribution of the study participants showed that about $36 \%$ of them were below 50 years of age. The mean age of the study participants was 55.03 (SD \pm 11.13 ) years. Among the total respondents, $60.6 \%$ were females, $74.2 \%$ were married, $44 \%$ have had at least one child in the household, $93 \%$ were farmers, $80.5 \%$ did not have formal education (Table 1). The wealth index of the respondents is shown in Figure 3.

\section{Personal and environmental characteristics of study participants}


About $43 \%$ of study participants reported that they wash their face two times a day, $71 \%$ did not use soap when washing their faces at least once per day. Nearly $46 \%$ of respondents did not have toilet facilities; among 264 individuals who have had toilet facilities, only $6 \%$ had improved ones (Table 2).

\section{Medical and surgical characteristics of respondents}

From the total study participants, $295(60 \%)$ received the service at the health centre, $178(36.2 \%)$ at the health post, and the remaining 19(3.9\%) at the hospital. The study showed that among individuals who had postoperative Trichiasis, only $15(12.8 \%)$ had performed epilation after surgery. From those individuals who performed epilation practice, seven of them epilated monthly, five individuals once per two weeks and three individuals once per week. The majority of respondents (98\%) received the annual routine Zithromax campaign medication and about $92 \%$ of the study participants' families took this routine Zithromax campaign medication (Table 3).

\section{The Prevalence of Postoperative Trichiasis}

In this study, the prevalence of postoperative Trichiasis was $23.8 \%(95 \% \mathrm{Cl}=19.9-27.8)$. The majority of recurrence occurred in a single eye which was $48 \%$ right eye, $47 \%$ left eye, and $5 \%$ in both eyes.

\section{Factors associated with postoperative Trichiasis}

The bi-variable binary logistic regression showed that sex, age, marital status, responsibility in the household, occupation, education status, frequency of face washing, soap use at least once a day, availability of toilet, travel to get water access, the number of surgery, place of surgery, operated eye, medication following surgery, duration since last surgery, pre-operative epilation history, complication after surgery, and knowledge about trachoma/Trichiasis were statistically significant association with postoperative Trichiasis at $p$-value $<0.2$. These variables were candidates for the multivariable analysis.

Multiple binary logistic regression (multivariable analysis) showed that age, frequency of face washing, duration since last surgery, medication following surgery, complication after surgery, pre-operation epilation history, and knowledge about trachoma/Trichiasis were factors associated with postoperative Trichiasis, $\mathrm{P}<0.05$.

Participants aged 50 to 59 years were 3.34 times $(A O R=3.34,95 \% \mathrm{Cl}=1.38,8.1)$ more likely to develop postoperative Trichiasis compared to those who were $<50$ years old. Age group between 60 and 69 years were 3.24 times (AOR=3.24, $95 \% \mathrm{Cl}=1.38,7.61)$ more likely to have had postoperative Trichiasis compared with the reference group. Those participants $\geq 70$ years old were 6 times $(A O R=6.04,95 \% \mathrm{Cl}=2.23,16.41)$ more likely to have had postoperative Trichiasis compared with the same reference group (i.e. $<50$ years old).

An individual who washed his/her face twice a day was $75 \%(A O R=0.25,95 \% \mathrm{Cl}=0.13,0.47)$ less likely to develop postoperative Trichiasis compared with the one washing his/her face once a day. Similarly, an individual who washed his/her face three and above times per day was $90 \%$ less likely $(A O R=0.1,95 \% \mathrm{Cl}=0.04,0.25)$ to develop postoperative Trichiasis compared with the same reference group.

Those who had taken Azithromycin following surgery were $81 \%(A O R=0.19,95 \% \mathrm{Cl}=0.09,0.41)$ less likely to have postoperative Trichiasis than those who took Tetracycline eye ointment.

When the duration since last surgery increased by one year, postoperative Trichiasis was 1.7 times more likely to occur keeping other variables constant $(\mathrm{AOR}=1.7,95 \% \mathrm{Cl}=1.35,2.14)$. 
Respondents who had complications after surgery were 2.98 times $(\mathrm{AOR}=2.98,95 \% \mathrm{Cl}=1.24,7.2)$ more likely to have had postoperative Trichiasis compared with those who did not had complication keeping other variables constant. Individuals who had pre-operative epilation history were 2 times ( $\mathrm{AOR}=2.11,95 \% \mathrm{Cl}=1.14,3.9)$ more likely to develop postoperative Trichiasis compared with those who did not have history of pre-operative epilation keeping other variables constant.

\section{Discussion}

Based on our study the prevalence of postoperative Trichiasis was $23.8 \%$. This finding was almost consistent with the studies conducted in the Ethiopia Amhara Region, Nepal and Tanzania; where postoperative Trichiasis rate was $24.7 \%$, $25 \%$, and $27.9 \%$ respectively $(2,15,16)$. However, it is lower than other studies done in Tanzania where the postoperative Trichiasis rate was 31\%, Gambia 41\%, Oman 47.2\%, and Nepal 28.9\% (4,17-19). This difference might be due to the differences in a surgical procedure. The study of Tanzania, Nepal, and Vietnam was used other than the PLTR technique. A study in the West Gojam Zone, Ethiopia shows PLTR procedure has a lower recurrence rate (20). Also, the finding of this study was higher than most studies conducted in Ethiopia ranges from 8-19\% (14, 21-25), the Jigawa State of Nigeria 17.3\% (26), and Vietnam 10.8-15.9\% $(27,28)$. This difference might be because the small sample size of the study and differences in the study population in which some previous study excluded individuals with repeat surgery that might reduce the recurrence rate of Trichiasis.

The elderly age had a significant association with Trichiasis recurrence. As people gets older, the likely hood of having postoperative Trichiasis increases by threefold. This finding was similar to the studies conducted in West Gojjam Zone, Ethiopia(15,20), and in four districts of Vietnam(27). This might be because as the age gets older, the body's immune response may be decreased (29). Additionally, the elderly have usually the highest prevalence of trachoma that can be attributed to additional contracture of the tarsal tissues leading to recurrence of Trichiasis.

Face washing is one component of the WHO SAFE strategy to eliminate trachoma with direct relation to reducing Trichiasis recurrence. In this study, increased frequency of face washing was statistically significant preventive factor of postoperative Trichiasis. No previous study had investigated the association between frequency of face washing and Trichiasis recurrence. The association might be because individuals who wash their faces would less frequently affected by trachoma which is a measure cause of Trichiasis recurrence.

Medication following TT surgery was the factor that has a significant association with the outcome variable. In this study, Azithromycin was associated with significantly less postoperative Trichiasis rate compared with tetracycline. This result was similar to the study done in Wolayta Soddo Ethiopia (30) and Nepal (17). But another randomized controlled trial study conducted in the Gambia shows that azithromycin did not improve the outcome of Trichiasis surgery(31). The possible reason might be a difference in the study participants who received medication following surgery. In the above Gambian study, all patients received Tetracycline eye ointment twice a day for two weeks, and subject randomized to Azithromycin group also received a $1 \mathrm{~g}$ dose of Azithromycin. But in our study, the participants were taken either Tetracycline or Azithromycin. Treatment with Azithromycin is an important component of the WHO SAFE strategy to eliminate trachoma by breaking the cycle and spread of ocular infection.

This study shows that when the duration since the last surgery was longer, the risk of postoperative Trichiasis was increased. It was in line with two studies conducted in Oman that found the length of time since surgery was an important predictor of Trichiasis recurrence. In those studies, during the early postoperative period the recurrence was not significantly high $(18,32)$.. When the duration increased the possibility of an individual having postoperative Trichiasis might increase due to the progression of the cicatricial process. But it contradicted with the study conducted in rural kebeles of west Gojam Zone, Ethiopia which shows that when the time is long since surgery the risk of 
recurrence was decreased (15). This difference might be due to our study includes individuals who have up to six years of duration.

When an individual has a good knowledge of trachoma and Trichiasis, the odds of having postoperative Trichiasis were decreased by $76.5 \%$. This good knowledge might result in a positive effect on the prevention of trachoma and postoperative Trichiasis. The study conducted in Alaba District which is located in Southern Ethiopia on Trachomatous Trichiasis founds the negative effect of poor knowledge on Trachoma prevention (33). But previous studies that investigated factors associated with Trichiasis recurrence were not included this variable. The community knowledge about Trachoma is mandatory to eliminate blinding trachoma and to prevent postoperative Trichiasis.

Complication after TT surgery was another factor that has a significant association with postoperative Trichiasis. An individual who has complication following TT surgery were more likely to have postoperative Trichiasis compared with those who didn't have a complication. This finding was in line with the study conducted in rural villages in the West Gojjam Zone, Amhara Regional State (15). But the study conducted in the provinces of Thanh Hoa, Vinh Phuc, and Ha Nam of Vietnam complication had no association (27). This difference might be due to the reason that readjustment after surgery was allowed in the study of Vietnam, this makes a decrease in the severity of complications.

Epilation is used as a self-treatment method for individuals who have Trichiasis. In this study, pre-operative epilation history was found to be statistically significantly associated with postoperative Trichiasis. This finding was in line with the study conducted in Wolayta zone Southern Ethiopia (22). But another study in a similar study area was found epilation before surgery had no association with postoperative Trichiasis (34). This difference might be because epilation history was self-report and it might lead to misclassification. Repetitive epilation especially in rural areas the material might harm the matrix, the portion of the hair that grows actively, and follicular stem cells. Also, it makes the new hair to be sharp and the epidermis becomes less responsive possibly due to a decrease in the number of stimulusresponsive cells.

Limitations of the study

The study could not include some factors like pre-operative Trichiasis severity, incision length, and suture type which were impossible to find the data.

The other limitation was related to the development of Trichiasis. It was not known exactly when the individual developed Trichiasis since recurrence in the early postoperative period may occur for different reasons than late recurrence.

\section{Conclusions}

The prevalence rate of postoperative Trichiasis in the study area was higher than most Ethiopian studies. Our finding indicated that the risk of postoperative Trichiasis increased due to old age, long duration since surgery, pre-operative epilation history and complication after surgery. On the other hand, face washing more than one time per day, good knowledge about trachoma/Trichiasis and taking azithromycin medication following surgery decreased the rate of postoperative Trichiasis.

Improving the knowledge and health extension package especially personal hygiene and environmental sanitation in the community, properly training of IECWs, follow up visits to patients who have had TT surgery, early detection, and management of complications are important measures that need to be taken to decrease the rate of postoperative Trichiasis. 


\section{Acronyms And Abbreviations}

AOR Adjusted Odds Ratio

BTR Bi-lamellar Tarsal Rotation

COR Crude Odds Ratio

EFY Ethiopian Fiscal Year

IECWs Integrated Eye Care Workers

PLTR Posterior Lamellar Tarsal Rotation

SAFE Surgery Antibiotic Face cleanness Environmental improvement

SPSS Statistical Product and service solution

TF Trachomatous Inflammation, Follicular

TI Trachomatous Inflammation, Intense

TPR Tarsal Plate Rotation

TS Trachomatous Scaring

TT Trachomatous Trichiasis

TTC Tetracycline

WHO World Health Organization

\section{Declarations}

\section{Ethics approval and consent to participate}

Primarily, ethical clearance was obtained from the College of Medical and health science Institutional Review Board (IRB) Committee, of Bahir Dar University. Then, all participants were asked for their verbal consent to be part of the study after clear explanation about the purpose of the study. This was because most of the study participants were rural residents; that is, most of them couldn't read and write and they were familiar with the procedure since they had TT surgery. The Ethics committee approved obtaining verbal consent. Confidentiality was ensured throughout the process by coding. And also, data collectors were put their signature for they had verbal informed consent for the interview and the observation done.

\section{Consent for publication}

Not applicable

\section{Availability of data and material}

The datasets used and/or analyzed during the current study are available from the corresponding author on reasonable request. 


\section{Competing interests}

The authors declare that they have no competing interests.

\section{Funding}

No fund was received

\section{Authors' contributions}

AT conceived and designed the research protocol.

GT and GD approved the proposal with extensive revisions, participated in the data analysis, and had written the manuscript. All authors have read and approved the final manuscript.

\section{Acknowledgments}

The authors would like to thank, Bahir Dar University, the study participants who freely give their time and Amhara health bureau and Ambassel district health office for facilitating the study and their support.

\section{References}

1. Record WE. Weekly Epidemiological R. 2019, vol. 94, 16 [full issue]. Weekly Epidemiological Record = Relevé épidémiologique hebdomadaire. 2019;94(16):189 - 96.

2. West ES, Mkocha H, Munoz B, Mabey D, Foster A, Bailey R, et al. Risk factors for postsurgical trichiasis recurrence in a trachoma-endemic area. Investig Ophthalmol Vis Sci. 2005;46(2):447-53.

3. Courtright P. Acceptance of surgery for trichiasis among rural Malawian women. East Afr Med J. 1994;71(12):803-4.

4. Rajak SN, Makalo P, Sillah A, Holland MJ, Mabey DC, Bailey RL, et al. Trichiasis surgery in The Gambia: a 4-year prospective study. Investig Ophthalmol Vis Sci. 2010;51(10):4996-5001.

5. Hr W, Turner A, Hr T, Trachoma. Lancet. 2008;371(9628):1945-54.

6. Center TC. Summary proceedings Nineteenth Annual Trachoma Program Review Celebrating 20 Years of Impact. March 19-20, 2018.

7. Mariotti SP, Pascolini D, Rose-Nussbaumer J. Trachoma: global magnitude of a preventable cause of blindness. $\mathrm{Br}$ J Ophthalmol. 2009;93(5):563-8.

8. Pascolini D, Mariotti SP. Global estimates of visual impairment: 2010. Br J Ophthalmol. 2012;96(5):614-8.

9. WHO

WHO. Trachoma. 2019, [cited 201902 Dec]. Avilable https://www.who.int/news-room/factsheets/detail/trachoma. 2019.

10. London School of Hygiene \& Tropical Medicine.

Learn F. https://www.futurelearn.com/courses/eliminating-trachoma/0/steps/21665. London School of Hygiene \& Tropical Medicine.. 2020.

11. ICTC. Global Scientific Meeting on Trachomatous Trichiasis. https://wwwbicomalawiorg/manuals/_Global_Scientific_Meeting_on_Trachomatous_Trichiasispdf.. 2012.

12. Mwangi GW. Post-operative Trachomatous Trichiasis in Africa: a systematic review and online survey 2019.

13. office Awh. Annual report of non communicable diseases. 2019. 
14. Assefa Y, Habte D, Yigzaw T, Mekonnen A, Gebrie T, Dubale T, et al. Trichiasis ercurrence in Nother Ethiopia: a one year prospecitve study of Trachomatous Trichiasis surgery done by integrated eye care workers. Ethiopian Journal of Health Development. 2008;22(1):8-13.

15. Rajak SN, Habtamu E, Weiss HA, Kello AB, Abera B, Zerihun M, et al. The outcome of trachomatous trichiasis surgery in Ethiopia: risk factors for recurrence. PLoS neglected tropical diseases. 2013;7(8).

16. Zhang H, Kandel RP, Sharma B, Dean D. Risk factors for recurrence of postoperative trichiasis: implications for trachoma blindness prevention. Arch Ophthalmol. 2004;122(4):511-6.

17. Zhang H, Kandel RP, Atakari HK, Dean D. Impact of oral azithromycin on recurrence of trachomatous trichiasis in Nepal over 1 year. British journal of ophthalmology. 2006;90(8):943-8.

18. Khandekar R, Mohammed AJ, Courtright P. Recurrence of trichiasis: a long-term follow-up study in the Sultanate of Oman. Ophthalmic Epidemiol. 2001;8(2-3):155-61.

19. Merbs SL, Oktavec KC, Munoz BE, Perlini EF, West SK, Othman MS, et al. Lower postoperative scar height is associated with increased postoperative trichiasis 1 year after bilamellar tarsal rotation surgery. Ophthalmic Epidemiol. 2015;22(3):200-7.

20. Habtamu E, Wondie T, Aweke S, Tadesse Z, Zerihun M, Gashaw B, et al. Predictors of trachomatous trichiasis surgery outcome. Ophthalmology. 2017;124(8):1143-55.

21. Pearson K, Habte D, Zerihun M, King JD, Gebre T, Emerson PM, et al. Evaluation of community-based trichiasis surgery in Northwest Ethiopia. Ethiopian journal of health sciences. 2013;23(2):131-40.

22. Talero SL, Muñoz B, West SK. Potential Effect of Epilation on the Outcome of Surgery for Trachomatous Trichiasis. Translational vision science technology. 2019;8(4):30-.

23. Woreta F, Munoz B, Gower E, Alemayehu W, West SK. Three-year outcomes of the surgery for trichiasis, antibiotics to prevent recurrence trial. Archives of ophthalmology. 2012;130(4):427-31.

24. Habtamu E, Wondie T, Aweke S, Tadesse Z, Zerihun M, Zewudie Z, et al. Posterior lamellar versus bilamellar tarsal rotation surgery for trachomatous trichiasis in Ethiopia: a randomised controlled trial. The Lancet Global Health. 2016;4(3):e175-e84.

25. Rajak SN, Habtamu E, Weiss HA, Kello AB, Gebre T, Genet A, et al. Absorbable versus silk sutures for surgical treatment of trachomatous trichiasis in Ethiopia: a randomised controlled trial. PLoS medicine. 2011;8(12).

26. Ramyil A, Bascaran C, Bunce C, Alada J, Wade P, Mpyet C. Outcome of trachoma lid surgeries in Jigawa state, Nigeria. Cogent Medicine. 2016;3(1):1233683.

27. Thanh T, Khandekar R, Luong V, Courtright P. One year recurrence of trachomatous trichiasis in routinely operated Cuenod Nataf procedure cases in Vietnam. British journal of ophthalmology. 2004;88(9):1114-8.

28. Khandekar R, Thanh TTK, Luong VQ. The determinants of trichiasis recurrence differ at one and two years following lid surgery in Vietnam: A community-based intervention study. Oman journal of ophthalmology. 2009;2(3):119.

29. Gomez CR, Nomellini V, Faunce DE, Kovacs EJ. Innate immunity and aging. Experimental gerontology. 2008;43(8):718-28.

30. West S, Alemayehu W, Munoz B, Gower EW. Azithromycin prevents recurrence of severe trichiasis following trichiasis surgery: STAR trial. Ophthalmic Epidemiol. 2007;14(5):273-7.

31. Burton M, Kinteh F, Jallow O, Sillah A, Bah M, Faye M, et al. A randomised controlled trial of azithromycin following surgery for trachomatous trichiasis in the Gambia. Br J Ophthalmol. 2005;89(10):1282-8.

32. Khandekar R, Al-Hadrami K, Sarvanan N, Al Harby S, Mohammed AJ. Recurrence of trachomatous trichiasis 17 years after bilamellar tarsal rotation procedure. Am J Ophthalmol. 2006;141(6):1087-91. e1. 
33. Wondimu A, Bejiga A. Prevalence of trachomatous trichiasis in the community of Alaba District, Southern Ethiopia. East Afr Med J. 2003;80(7):365-9.

34. Gower EW, Merbs SL, Munoz BE, Kello AB, Alemayehu W, Imeru A, et al. Rates and risk factors for unfavorable outcomes 6 weeks after trichiasis surgery. Investig Ophthalmol Vis Sci. 2011;52(5):2704-11.

\section{Tables}

Table 1: Socio-demographic characteristics of respondents in Ambassel District, South Wollo Zone, Northeast Ethiopia, 2020

$(N=492)$ 


\begin{tabular}{|c|c|c|c|}
\hline Variables & & Frequency & $\%$ \\
\hline \multirow[t]{4}{*}{ Age } & $<50$ & 175 & 35.6 \\
\hline & $50-59$ & 123 & 25 \\
\hline & $60-69$ & 147 & 29.9 \\
\hline & $\geq 70$ & 47 & 9.6 \\
\hline \multirow[t]{2}{*}{ Sex } & Male & 194 & 39.4 \\
\hline & Female & 298 & 60.6 \\
\hline \multirow[t]{4}{*}{ Marital status } & Single & 10 & 2.0 \\
\hline & Married & 365 & 74.2 \\
\hline & Divorced & 42 & 8.5 \\
\hline & Widowed & 75 & 15.2 \\
\hline \multirow[t]{2}{*}{ Responsibility in house } & Head & 446 & 90.7 \\
\hline & Other family members & 46 & 9.3 \\
\hline \multirow[t]{2}{*}{ Family size } & Family size $<4$ & 189 & 38.4 \\
\hline & Family size >=4 & 303 & 61.6 \\
\hline \multirow[t]{2}{*}{$<10$ yrs children in the household } & Yes & 217 & 44.1 \\
\hline & No & 275 & 55.9 \\
\hline \multirow[t]{2}{*}{ Occupation } & Farmer & 457 & 92.9 \\
\hline & Merchant & 35 & 7.1 \\
\hline \multirow[t]{2}{*}{ Education status } & No formal Education & 396 & 80.5 \\
\hline & Elementary & 96 & 19.5 \\
\hline \multirow[t]{2}{*}{ Residence } & Urban & 82 & 16.7 \\
\hline & Rural & 410 & 83.3 \\
\hline \multirow[t]{3}{*}{ Religion } & Orthodox & 270 & 54.9 \\
\hline & Muslim & 210 & 42.7 \\
\hline & Protestant & 12 & 2.4 \\
\hline
\end{tabular}

Table 2: Personal and environmental characteristics of respondents in Ambassel District, South Wollo, Northeast Ethiopia, 2020

$(\mathrm{N}=492)$ 


\begin{tabular}{|llll|}
\hline Variables & & Frequency & $\%$ \\
\hline Frequency of face washing & once a day & 137 & 27.8 \\
\cline { 2 - 4 } & twice a day & 213 & 43.3 \\
\cline { 2 - 4 } & three and above per day & 142 & 28.9 \\
\hline Soap use & Yes & 142 & 28.9 \\
\cline { 2 - 4 } & No & 350 & 71.1 \\
\hline Knowledge about trachoma & Good & 434 & 88.2 \\
\cline { 2 - 4 } & Poor & 35 & 7.1 \\
\hline Have a toilet & Yes & 264 & 53.7 \\
\hline Type of toilet & No & 228 & 46.3 \\
\hline \multirow{2}{*}{ Toilet utilization } & Improved & 16 & 6.1 \\
\hline Travel to get water & Unimproved & 248 & 93.9 \\
\hline & Yes & 251 & 95.1 \\
\hline & Some of the family & 13 & 4.9 \\
\hline & $<30$ minute & 235 & 47.8 \\
\hline
\end{tabular}

Table 3: Medical and surgical characteristics of study participants in Ambassel District, South Wollo Zone, Northeast Ethiopia, 2020

$(\mathrm{N}=492)$ 


\begin{tabular}{|c|c|c|c|}
\hline Variables & & Frequency & $\%$ \\
\hline \multirow[t]{2}{*}{ Number of surgery } & One time & 409 & 83.1 \\
\hline & Two and above times & 83 & 16.9 \\
\hline \multirow[t]{3}{*}{ Eyelid operated } & Right & 131 & 26.6 \\
\hline & Left & 156 & 31.7 \\
\hline & Both & 205 & 41.7 \\
\hline \multirow[t]{2}{*}{ Medication is taken following surgery } & azithromycin/ oral tablet & 173 & 35.2 \\
\hline & tetracycline eye ointment & 319 & 64.8 \\
\hline \multirow[t]{6}{*}{ Duration since last surgery in years } & One year & 39 & 7.9 \\
\hline & Two years & 134 & 27.2 \\
\hline & Three years & 137 & 27.8 \\
\hline & Four years & 81 & 16.5 \\
\hline & Five years & 60 & 12.2 \\
\hline & Six years & 41 & 8.3 \\
\hline \multirow[t]{2}{*}{ History of epilation before surgery } & Yes & 150 & 30.5 \\
\hline & No & 342 & 69.5 \\
\hline \multirow[t]{3}{*}{ Frequency of epilation before surgery } & once a week & 52 & 10.6 \\
\hline & once per two week & 48 & 9.8 \\
\hline & once per month & 50 & 10.2 \\
\hline \multirow[t]{2}{*}{ Complications after surgery } & Yes & 46 & 9.3 \\
\hline & No & 446 & 90.7 \\
\hline \multirow[t]{7}{*}{ Surgeons code who perform this operation } & $A$ & 72 & 14.6 \\
\hline & B & 94 & 19.1 \\
\hline & C & 74 & 15.0 \\
\hline & $\mathrm{D}$ & 74 & 15.0 \\
\hline & $\mathrm{E}$ & 101 & 20.5 \\
\hline & $\mathrm{F}$ & 61 & 12.4 \\
\hline & Other hospital nurses & 16 & 3.3 \\
\hline \multirow[t]{2}{*}{ Face cleanliness } & Yes & 421 & 86.4 \\
\hline & No & 71 & 14.4 \\
\hline \multirow[t]{2}{*}{ Current trachoma status } & Yes & 154 & 31.3 \\
\hline & No & 338 & 68.7 \\
\hline
\end{tabular}


Table 4: Multi-variable analysis of binary logistic regression model for various factors and postoperative Trichiasis in Ambassel District, South Wollo Zone, Northeast Ethiopia, 2020 


\begin{tabular}{|c|c|c|c|c|c|c|}
\hline \multirow[t]{2}{*}{ Variables } & & \multicolumn{2}{|c|}{ Postoperative outcome } & \multirow[t]{2}{*}{$\operatorname{COR}(95 \% \mathrm{Cl})$} & \multirow[t]{2}{*}{$\mathrm{AOR}(95 \% \mathrm{Cl})$} & \multirow[t]{2}{*}{ P-value } \\
\hline & & Recurrence & $\begin{array}{l}\text { No } \\
\text { recurrence }\end{array}$ & & & \\
\hline \multirow[t]{4}{*}{ Age } & $<50$ & 11 & 164 & 1.00 & 1.00 & \\
\hline & $50-59$ & 23 & 100 & $\begin{array}{l}3.43(1.6 \\
-7.34)\end{array}$ & $\begin{array}{l}3.34(1.38- \\
8.1)\end{array}$ & 0.007 \\
\hline & $60-69$ & 57 & 90 & $\begin{array}{l}9.44(4.71- \\
18.92)\end{array}$ & $\begin{array}{l}3.24(1.38- \\
7.61)\end{array}$ & 0.007 \\
\hline & $>=70$ & 26 & 21 & $\begin{array}{l}18.46(7.98- \\
42.7)\end{array}$ & $\begin{array}{l}6.04(2.25- \\
16.41)\end{array}$ & $<0.000$ \\
\hline \multirow[t]{2}{*}{ Sex of Respondent } & Male & 40 & 154 & $\begin{array}{l}0.75(0.48- \\
1.15)\end{array}$ & $\begin{array}{l}0.72(0.38- \\
1.36)\end{array}$ & 0.312 \\
\hline & Female & 77 & 221 & 1 & 1 & \\
\hline \multirow[t]{4}{*}{ marital status } & Single & 0 & 10 & 0 & 0 & .999 \\
\hline & Married & 73 & 292 & $\begin{array}{l}0.27(0.16- \\
0.46)\end{array}$ & $\begin{array}{l}1.34(0.59- \\
3.04)\end{array}$ & .488 \\
\hline & Divorced & 8 & 34 & $\begin{array}{l}0.5(0.11- \\
0.62)\end{array}$ & $\begin{array}{l}0.5(0.13- \\
1.85)\end{array}$ & .297 \\
\hline & Widowed & 36 & 39 & 1 & 1 & \\
\hline \multirow[t]{2}{*}{$\begin{array}{l}\text { Status/responsibility } \\
\text { in the house }\end{array}$} & Head & 98 & 348 & $\begin{array}{l}0.4(0.22- \\
0.75)\end{array}$ & $\begin{array}{l}1.15(0.38- \\
3.49)\end{array}$ & 0.807 \\
\hline & $\begin{array}{l}\text { Other family } \\
\text { members }\end{array}$ & 19 & 27 & 1 & 1 & \\
\hline \multirow[t]{2}{*}{$\begin{array}{l}\text { Occupation of the } \\
\text { respondent }\end{array}$} & Farmer & 112 & 345 & $\begin{array}{l}1.95(0.74- \\
5.14)\end{array}$ & $\begin{array}{l}0.83(0.18- \\
3.84)\end{array}$ & 0.815 \\
\hline & Merchant & 5 & 30 & 1 & 1 & \\
\hline \multirow[t]{2}{*}{ Educational status } & $\begin{array}{l}\text { No formal } \\
\text { Education }\end{array}$ & 107 & 289 & $\begin{array}{l}3.18(1.6- \\
6.36)\end{array}$ & $\begin{array}{l}1.45(0.48- \\
4.37)\end{array}$ & 0.506 \\
\hline & Elementary & 10 & 86 & 1 & 1 & \\
\hline \multirow{3}{*}{$\begin{array}{l}\text { Frequency of face } \\
\text { washing }\end{array}$} & Once a day & 68 & 69 & 1 & 1 & \\
\hline & Twice a day & 37 & 176 & $\begin{array}{l}0.22(0.13- \\
0.35)\end{array}$ & $\begin{array}{l}0.25(0.13- \\
0.47)\end{array}$ & $<0.000$ \\
\hline & $\begin{array}{l}\text { Three and } \\
\text { above per day }\end{array}$ & 12 & 130 & $\begin{array}{l}0.1(0.05- \\
0.19)\end{array}$ & $\begin{array}{l}0.1(0.04- \\
0.25)\end{array}$ & $<0.000$ \\
\hline \multirow[t]{2}{*}{ Soap use } & Yes & 21 & 121 & $\begin{array}{l}0.46(0.27- \\
0.77)\end{array}$ & $\begin{array}{l}0.76(0.36- \\
1.59)\end{array}$ & 0.469 \\
\hline & No & 96 & 254 & 1 & 1 & \\
\hline \multirow[t]{2}{*}{ Have a toilet } & Yes & 46 & 217 & $\begin{array}{l}0.47(0.31- \\
072)\end{array}$ & $\begin{array}{l}0.76(0.4- \\
1.45)\end{array}$ & 0.409 \\
\hline & No & 71 & 158 & 1 & 1 & \\
\hline Travel to get water & $<30$ minute & 50 & $\begin{array}{l}185 \\
\text { e } 19 / 22\end{array}$ & $0.77(0.50-$ & $0.94(0.5-$ & 0.835 \\
\hline
\end{tabular}




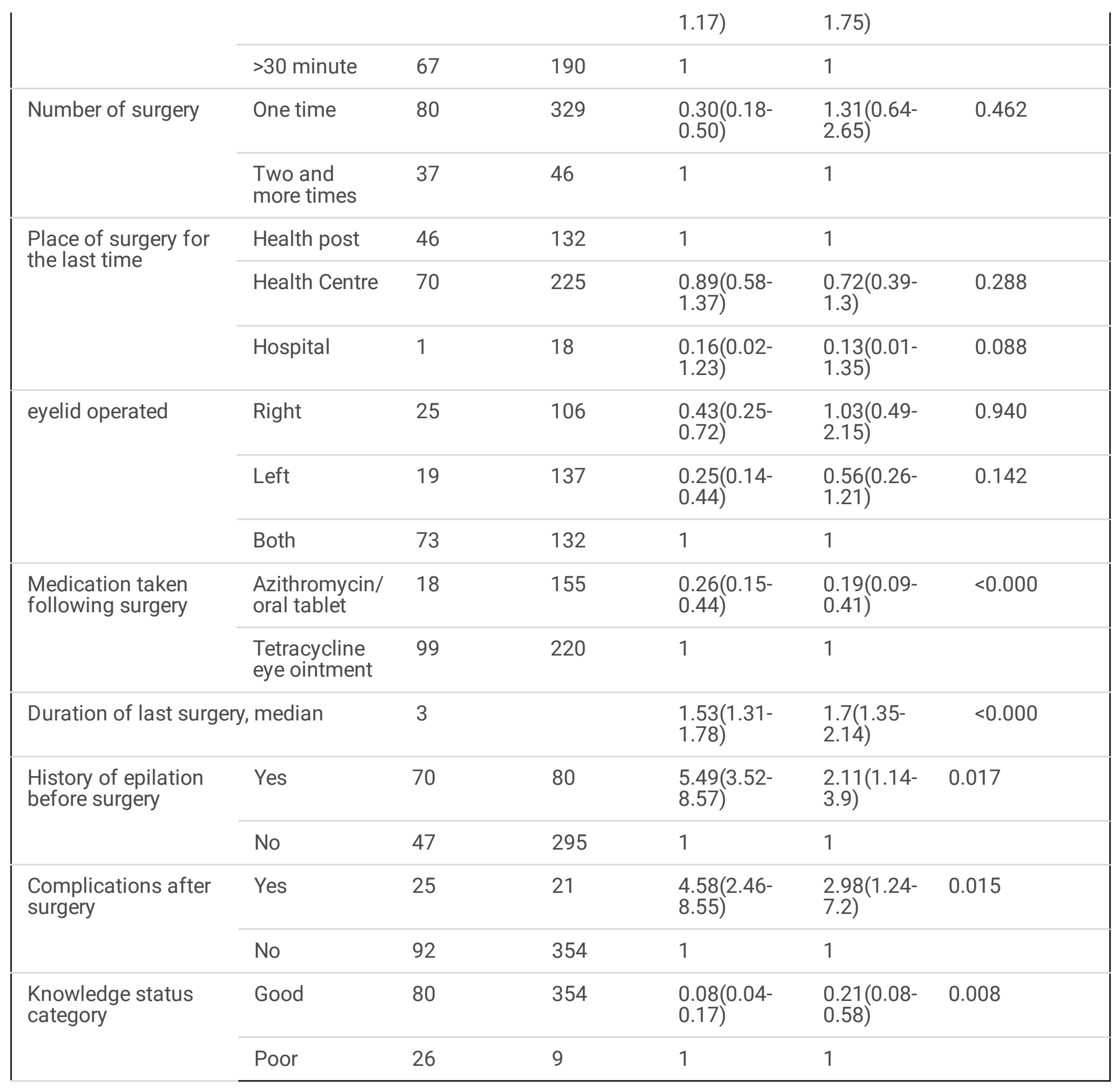

N.B Hosmer and Lemeshow test $=0.975$

$\mathrm{SE}=$ all variables are $<0.6$

\section{Figures}




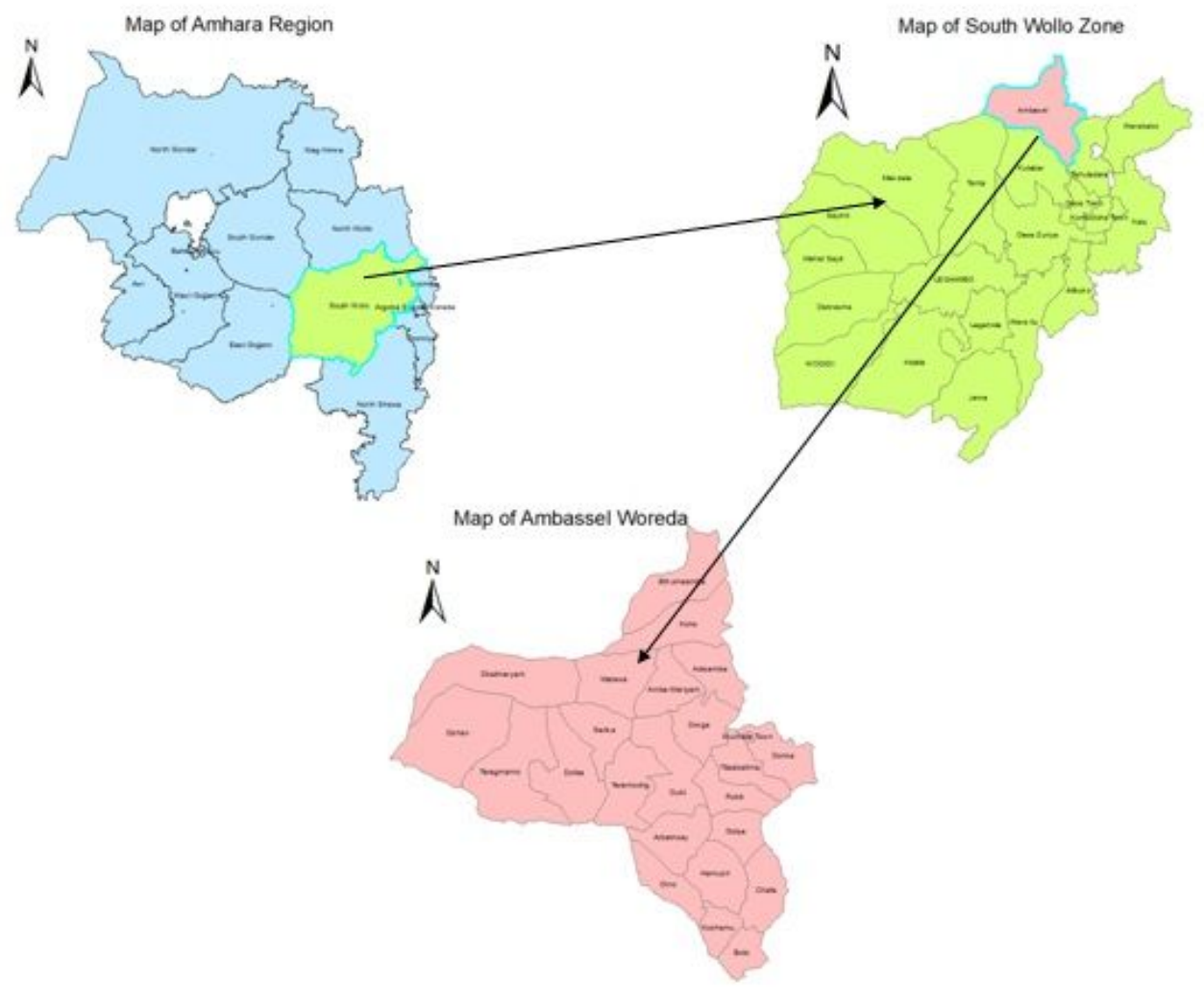

Figure 1

Administration map of Ambassel District, South Wollo Zone, and North East Ethiopia, prepared by using ArcGIS, 2020.

\section{Schematic presentation of sampling}

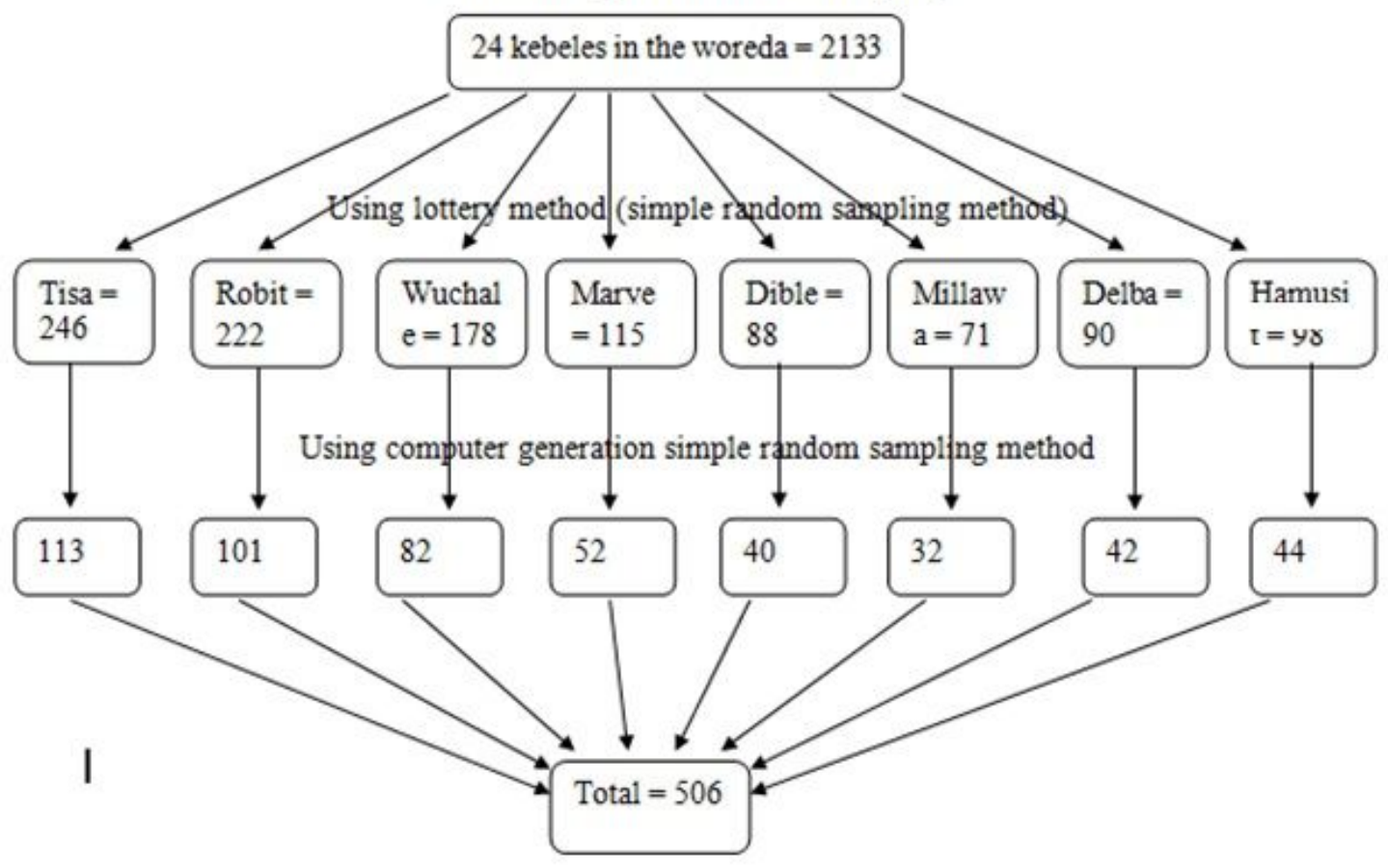

\section{Figure 2}

Schematic presentation of the sampling procedure, in Ambassel District, South Wollo Zone, Northeast Ethiopia, 2020. 


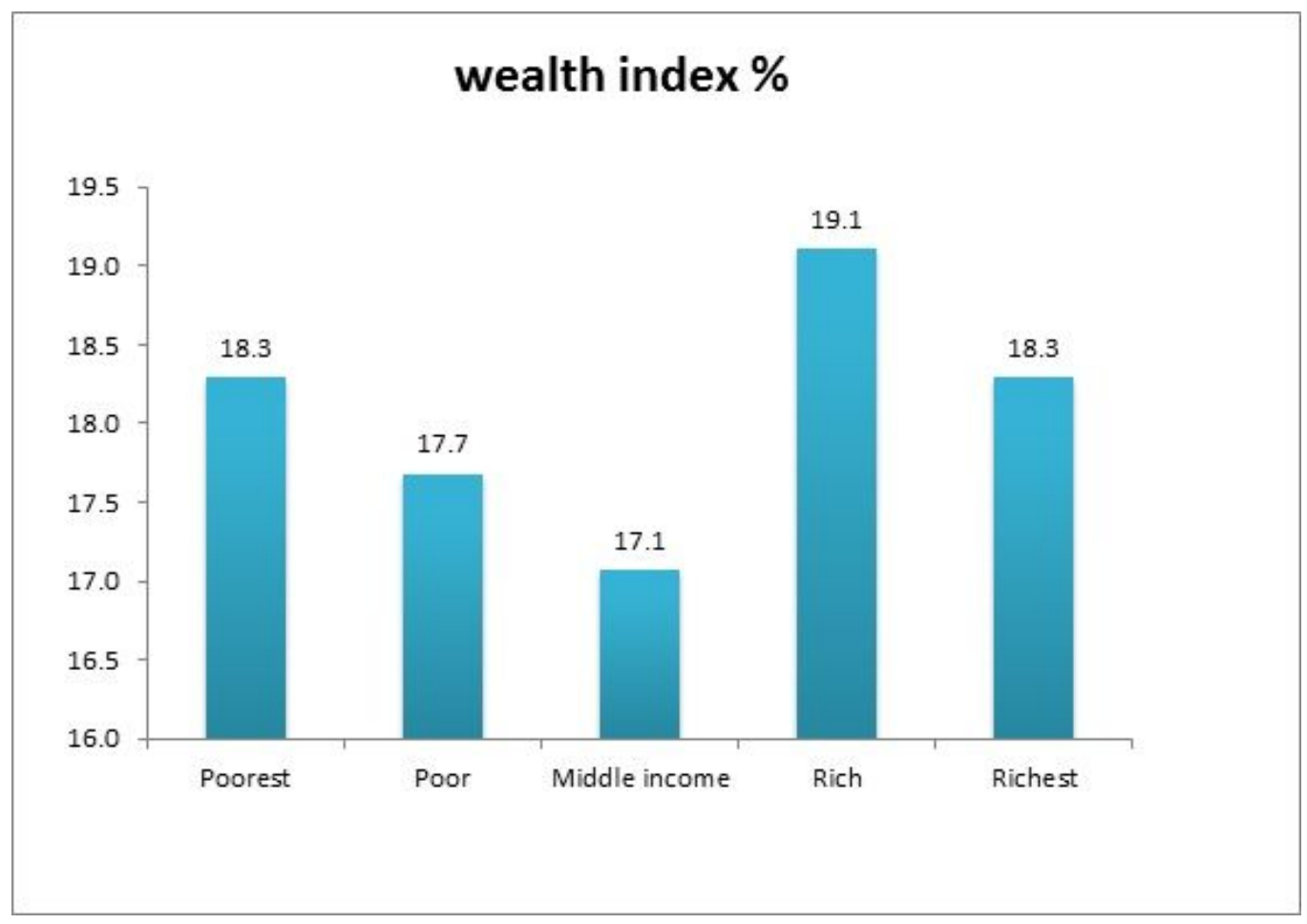

Figure 3

Wealth index of respondents in Ambassel District, South Wollo Zone, Northeast Ethiopia, 2020. 